\title{
Comparative Analysis of Food Composition and Mineral Element Levels of Two Locally Prepared Foods in Jigawa State Nigeria
}

\author{
Fatima Ibrahim Baiwa ${ }^{1}$, Abdulhadi Yakubu ${ }^{1}$, Garba Uba Kanya ${ }^{1}$, \\ Salisu Ahmed ${ }^{1}$ and Shuaib Nasir Halliru ${ }^{2}$ \\ ${ }^{1}$ Deparment of Science Laboratory Technology, College of Science and Technology, \\ Jigawa State Polytechnic Dutse, Nigeria \\ ${ }^{2}$ Department of Biology, Sa'adatu Rimi College of Education Kumbotso, Kano Nigeria
}

*Corresponding author

Ke y w o r d s
$\begin{aligned} & \text { Comparative } \\ & \text { analysis, Food } \\ & \text { Composition and } \\ & \text { Mineral level }\end{aligned}$
Article Info
$\begin{aligned} & \text { Accepted: } \\ & \text { 17 December } 2018 \\ & \text { Available Online: } \\ & \text { 10 January } 2019\end{aligned}$

\section{Introduction}

In recent years, consumers have shown an increased interest and demand for traditional foods as they are often perceived as having specific sensory characteristics and being of higher quality. European Food Information Resource (EuroFIR) defined traditional foods as a food of specific feature or features, which
The use of cereal grains as sources of food for man is well known. Different food products are obtained from grains based on the processing technique and the type of cereal employed. Although the causes of malnutrition are many and diverse, inadequate intake of foods and essential nutrients is a major contributory factor and yet this subject is poorly researched in many developing countries including Nigeria. This research was conducted in Jigawa State Nigeria. Were a total of 1,500 questionnaires were administered and 1,250 retrieved. Based on the analysed questionnaires, two representative samples of traditional foods which include Tuwon Masara (white maize) and Danwake were collected and transported to the laboratory for chemical food composition and mineral elements analysis. Standard procedures of AOAC were used to determine the carbohydrate content, crude protein, crude fat, ash, moisture content, total fiber, energy ( $\mathrm{kcal}$ ), glycosides, oxalate, phytate, tannin, total phenol as well as some micro and macro elements. From the result obtained, Tuwon masara was found to have a high percentage of both chemical food composition and mineral elements than Danwake. With respect to standard by Recommended Dietary Allowance (DRA) of Food and Agricultural Organization (FAO), both foods have high chemical food composition but lower mineral element levels. This may be attributed to lack of standard procedure on the actual amount of nutrients for the

distinguish it clearly from other similar products of the same category in terms of the use of 'traditional ingredients' (raw materials or primary products) or 'traditional composition' or 'traditional type of production and processing method", (Trichopoulou et al., 2007). Humans require more than 22 mineral elements, which can all be supplied by an appropriate diet. However, 
the diets of populations subsisting on cereals, or inhabiting regions where soil mineral imbalances occur, often lack Fe, $\mathrm{Zn}, \mathrm{Ca}, \mathrm{Mg}$, $\mathrm{Cu}$, I or Se. Some are required in large amounts, but others, such as $\mathrm{Fe}, \mathrm{Zn}, \mathrm{Cu}$, I and $\mathrm{Se}$, are required in trace amounts because higher concentrations can be harmful (Grusak and Cakmak, 2005). Ultimately, these mineral elements enter the food chain through plants while some essential mineral elements, such as $\mathrm{K}$ and $\mathrm{Na}$, occur solely as soluble inorganic ions in plants. The mineral elements most frequently lacking in human diets are $\mathrm{Fe}, \mathrm{Zn}$ and I, although other elements, such as $\mathrm{Ca}$, $\mathrm{Mg}, \mathrm{Cu}$ and $\mathrm{Se}$, can be deficient in the diets of some populations (White and Broadley, 2005). These deficiencies are caused by diets characterized by high intakes of staple foods but low intakes of vegetables, fruits, and animal and fish products, which are rich sources of minerals.

The use of cereal grains as sources of food for man is well known. Different food products are obtained from grains based on the processing technique and the type of cereal employed. Developed countries utilize up to $80 \%$ of their available cereals as animal feed. The trend is different in African countries where traditional human foods are based on millet, maize and rice. Legumes are, in most cases, supplemented to complement proteins in some of the preparations. Some traditional foods in Nigeria made from cereals include Tuwo, Danwake Ogi, Kunu, Burukutu and Pito (Okafor, 1983). Tuwo and Danwake are some of the most commonly used cereal food produced from maize and millet respectively.

They are some of the most common foods used by the people of northern Nigeria and some other parts of the country (Gaffa et al. 2002). Lack of standard procedure for the processing of these local foods may lead to malnutrition which can be brought about by the inadequacy or over-consumption of one or more of the essential nutrients necessary for survival, growth and reproduction, as well as productivity at work (UNICEF, 2009). Micronutrient malnutrition greatly increases mortality and morbidity rates, diminishes cognitive abilities of children and lowers their educational attainment, reduces labor productivity, stagnates national development efforts, contributes to continued high population growth rates and reduces the livelihood and quality of life for all those affected (Welch and Graham, 1999). Although the causes of malnutrition are many and diverse, inadequate intake of foods and essential nutrients is a major contributory factor and yet this subject is poorly researched in many developing countries including Nigeria (Kikafunda et al., 2006). As such, this research will provide a comparative study of food composition and mineral element levels of two traditional foods consumed in Jigawa State Nigeria which may help not only to identify the nutritional status of local diets, but may also lead to effective strategies being developed for the purpose of enhancing the nutrient composition of these traditional diets (Welch and Graham, 2002).

\section{Study area}

Jigawa State is in the North western part of Nigeria between latitudes $11.00^{\circ} \mathrm{N}$ to $13.00^{\circ} \mathrm{N}$ and longitudes $8.00^{\circ} \mathrm{E}$ to $10.15^{\circ} \mathrm{E}$ and has a total population of 4,348,649 inhabitants (NPC, 2006). About 80 per /cent of the population is found in the rural areas and predominately farmers and Muslims. The socio-cultural situation in Jigawa State could be described as homogeneous: it is mostly populated by Hausa/Fulani, who can be found in all parts of the State.

\section{Sample size and sample collection}

A mixed questionnaire (open and closed) in both local language (Hausa) and English were 
used as an instrument for data collection. A clustered sampling method was strictly adhered to, in which the whole Jigawa state was divided into three clusters based on the three senatorial districts of the state. A total of 1,500 questionnaires were administered to the population of 4,348,649 (500 to each senatorial district) in which 1,250 were returned. Based on the filled questionnaires, two representative samples of traditional foods which include Tuwon Masara and Danwake were selected, collected and transported to the laboratory for chemical food composition analysis.

\section{Food composition}

Food composition of locally prepared Tuwon masara (maize) with Kuka (baobab leaves) soup and Danwake. For Tuwon masara, ingredientsinclude maize (Masara), baobab leaves $(k u k a)$, African locust bean seeds (daddawa) and meat. Danwake have ingredients such as beans (wake), Groundnut oil, Guinea corn (dawa), pepper, baobab leaves $(k u k a)$, potash and cassava. For comparison purposes, standard quantity of some nutritional contents and mineral element levels of the recipes from Food and Agricultural Organisation (FAO) of the United Nations were presented.

\section{Chemical analyses}

Standard procedures of AOAC were used to determine the carbohydrate content, crude protein, crude fat, ash, moisture content, total fiber, energy (kcal), glycosides, oxalate, phytate, tannin and total phenol as well as all the mineral elements (AOAC, 1990). Energy value was calculated using the Atwater's conversion factors. Minerals were determined by a Zeeman Polarized Atomic Absorption Spectrophotometer, Hitachi Model 180-80, and Ion Chromatographic Analyzer ICA model IC 100 (Spackman and Stein, 1958). All reagents for the analysis of food are of analytical grade according to the specification of the manufacturers

\section{Statistical analysis}

Results were expressed as mean \pm standard deviation. The difference between groups of each parameter was determined using the $\mathrm{t}-$ test and statistical significance were claimed at $\mathrm{P}<0.05$.

\section{Results and Discussion}

The proximate nutrient composition of most prepared local diet called white maize (Tuwon masara) and its recipes as well as their corresponding mineral elements are presented in Table 1 and 2 . The results indicated that total carbohydrate, crude protein, fiber, fat, moisture and energy values are higher than what is recommended by Dietary Recommended Allowance value (DRA). Other recipes such as tannin, glycosidase, ash content, total phenol, oxalate and phytate indicate a high value from most prepared food (Tuwon masara) collected from selected sites than the one prepared in the laboratory. From table 2, certain important mineral elements such as magnesium $(\mathrm{Mg})$, manganese $(\mathrm{Mn})$, iron $(\mathrm{Fe})$, lead $(\mathrm{Pb})$, Potassium $(\mathrm{K})$ Calcium $(\mathrm{Ca})$, Cupper $(\mathrm{Cu})$, Cobalt $(\mathrm{Cb})$ and other mineral elements were analysed.

Table 3 shows the percentage Proximate and Anti Nutritional Content of Recipe for Moderately Selected Diet (Danwake Served with Groundnut Oil and Pepper) in (g/100g) and the percentage proximate consumed in Jigawa State, Nigeria. From this table, it was also observed that there is a high percentage of carbohydrate, crude protein, fiber, fat, moisture and energy values than the prepared food in the laboratory (Table 4) as well as what is recommended by Dietary Recommended Allowance value (DRA) in Table 5. 
Table.1 Percentage (g/100g) proximate and Anti-Nutritional Contents of Recipe for Tuwon Masara (white maize) Served with Kuka Soup Consumed in Jigawa State, Nigeria

\begin{tabular}{|c|c|c|c|c|c|c|c|c|c|c|c|c|}
\hline $\begin{array}{l}\text { Parameters } \\
\text { Sample }\end{array}$ & Carbohydrate & $\mathrm{C} / \mathrm{P}$ & C/Fat & $\mathbf{C} / \mathbf{F}$ & Moisture & Ash & $\begin{array}{l}\text { Energy } \\
\text { (kcal) }\end{array}$ & $\begin{array}{l}\text { C. } \\
\text { glycosides } \\
(\mathrm{mg} / 100 \mathrm{~g})\end{array}$ & $\begin{array}{l}\text { Oxalate } \\
(\mathrm{mg} / 100 \mathrm{~g})\end{array}$ & Phytate & Tannin & $\mathbf{T} / \mathbf{P}$ \\
\hline $\begin{array}{l}\text { Maize } \\
\text { (white) }\end{array}$ & $\begin{array}{c}84.0300 \\
\pm \\
1.4042\end{array}$ & $\begin{array}{c}8.4778 \\
\pm \\
1.2945\end{array}$ & $\begin{array}{c}0.2764 \\
\pm \\
0.0022\end{array}$ & $\begin{array}{c}0.3617 \\
\pm \\
0.0823\end{array}$ & $\begin{array}{c}6.5000 \\
\pm \\
0.2236\end{array}$ & $\begin{array}{c}0.2542 \\
\pm \\
0.0340\end{array}$ & $\begin{array}{c}372.1667 \\
\pm \\
0.9458\end{array}$ & $\begin{array}{c}0.1250 \\
\pm \\
0.0010\end{array}$ & $\begin{array}{c}3.2633 \\
\pm \\
0.1050\end{array}$ & $\begin{array}{c}1.1951 \\
\pm \\
0.0177\end{array}$ & $\begin{array}{c}0.1602 \\
\pm \\
0.0049\end{array}$ & $\begin{array}{c}14.6849 \\
\pm \\
1.4585\end{array}$ \\
\hline Baobab & $\begin{array}{c}63.0754 \\
\pm \\
2.7174\end{array}$ & $\begin{array}{c}17.6889 \\
\pm \\
2.3411\end{array}$ & $\begin{array}{c}0.2762 \\
\pm \\
0.0054\end{array}$ & $\begin{array}{c}10.4975 \\
\pm \\
0.7862\end{array}$ & $\begin{array}{c}6.3333 \\
\pm \\
0.4216\end{array}$ & $\begin{array}{c}2.2296 \\
\pm \\
0.0187\end{array}$ & $\begin{array}{c}325.6667 \\
\pm \\
3.1269\end{array}$ & $\begin{array}{c}0.4220 \\
\pm \\
0.0130\end{array}$ & $\begin{array}{c}7.8833 \\
\pm \\
0.0883\end{array}$ & $\begin{array}{c}1.6089 \\
\pm \\
0.0496\end{array}$ & $\begin{array}{c}0.5059 \\
\pm \\
0.0066\end{array}$ & $\begin{array}{c}36.8128 \\
\pm \\
1.0375\end{array}$ \\
\hline Potash & $\begin{array}{c}17.4949 \\
\pm \\
0.1083\end{array}$ & $\begin{array}{c}1.6722 \\
\pm \\
0.0717\end{array}$ & $\begin{array}{c}0.2969 \\
\pm \\
0.0120\end{array}$ & $\begin{array}{c}0.6142 \\
\pm \\
0.0266\end{array}$ & $\begin{array}{c}6.3333 \\
\pm \\
0.3333\end{array}$ & $\begin{array}{c}73.7385 \\
\pm \\
0.2955\end{array}$ & $\begin{array}{c}79.3405 \\
\pm \\
0.8280\end{array}$ & $\begin{array}{c}0.1130 \\
\pm \\
0.0000\end{array}$ & $\begin{array}{c}0.9900 \\
\pm \\
0.0942\end{array}$ & $\begin{array}{c}0.4061 \\
\pm \\
0.0310\end{array}$ & $\begin{array}{c}0.0281 \\
\pm \\
0.0038\end{array}$ & $\begin{array}{c}0.3364 \\
\pm \\
0.0213\end{array}$ \\
\hline Onion & $\begin{array}{c}72.6134 \\
\pm \\
1.3581\end{array}$ & $\begin{array}{c}4.1222 \\
\pm \\
0.2144\end{array}$ & $\begin{array}{c}0.3056 \\
\pm \\
0.0139\end{array}$ & $\begin{array}{c}11.8175 \\
\pm \\
1.4136 \\
\end{array}$ & $\begin{array}{c}10.6667 \\
\pm \\
0.2108\end{array}$ & $\begin{array}{c}0.7079 \\
\pm \\
0.0305\end{array}$ & $\begin{array}{c}309.8333 \\
\pm \\
5.6179\end{array}$ & $\begin{array}{c}0.5340 \\
\pm \\
0.0040\end{array}$ & $\begin{array}{c}45.7600 \\
\pm \\
0.8271\end{array}$ & $\begin{array}{c}2.8155 \\
\pm \\
0.1947\end{array}$ & $\begin{array}{c}0.3783 \\
\pm \\
0.0187\end{array}$ & $\begin{array}{c}10.3760 \\
\pm \\
0.5955\end{array}$ \\
\hline Daddawa & $\begin{array}{c}41.8349 \\
\pm \\
5.4149\end{array}$ & $\begin{array}{c}30.6833 \\
\pm \\
5.4435\end{array}$ & $\begin{array}{c}0.2702 \\
\pm \\
0.0012\end{array}$ & $\begin{array}{c}14.9725 \\
\pm \\
0.1905\end{array}$ & $\begin{array}{c}11.0000 \\
\pm \\
0.0000\end{array}$ & $\begin{array}{c}1.2057 \\
\pm \\
0.0351\end{array}$ & $\begin{array}{c}292.5000 \\
\pm \\
1.1475\end{array}$ & $\begin{array}{c}0.1680 \\
\pm \\
0.0020\end{array}$ & $\begin{array}{c}22.2200 \\
\pm \\
1.0303\end{array}$ & $\begin{array}{c}1.1448 \\
\pm \\
0.0272\end{array}$ & $\begin{array}{c}0.3478 \\
\pm \\
0.0135\end{array}$ & $\begin{array}{c}16.1065 \\
\pm \\
0.0288\end{array}$ \\
\hline Meat & $\begin{array}{c}30.4242 \\
\pm \\
6.9736\end{array}$ & $\begin{array}{c}57.4333 \\
\pm \\
9.2501\end{array}$ & $\begin{array}{c}0.2784 \\
\pm \\
0.0101\end{array}$ & $\begin{array}{c}3.3071 \\
\pm \\
2.7349\end{array}$ & $\begin{array}{c}7.5714 \\
\pm \\
0.4286\end{array}$ & $\begin{array}{c}0.8998 \\
\pm \\
0.1018\end{array}$ & $\begin{array}{c}363.3333 \\
\pm \\
0.8028\end{array}$ & $\begin{array}{c}0.1490 \\
\pm \\
0.0010\end{array}$ & $\begin{array}{c}9.1300 \\
\pm \\
0.3150\end{array}$ & $\begin{array}{c}0.3558 \\
\pm \\
0.0285\end{array}$ & $\begin{array}{c}0.0978 \\
\pm \\
0.0075\end{array}$ & $\begin{array}{c}15.2998 \\
\pm \\
0.1255\end{array}$ \\
\hline Hot pepper & $\begin{array}{c}54.0580 \\
\pm \\
0.4917\end{array}$ & $\begin{array}{c}4.0600 \\
\pm \\
0.5351\end{array}$ & $\begin{array}{c}0.2949 \\
\pm \\
0.0122\end{array}$ & $\begin{array}{c}33.1200 \\
\pm \\
0.1420\end{array}$ & $\begin{array}{c}6.8000 \\
\pm \\
0.2000\end{array}$ & $\begin{array}{c}1.6671 \\
\pm \\
0.0132\end{array}$ & $\begin{array}{c}236.6667 \\
\pm \\
1.5635\end{array}$ & $\begin{array}{c}0.1970 \\
\pm \\
0.0130\end{array}$ & $\begin{array}{c}30.4700 \\
\pm \\
0.8814\end{array}$ & $\begin{array}{c}1.6437 \\
\pm \\
0.0202\end{array}$ & $\begin{array}{c}0.4230 \\
\pm \\
0.0162\end{array}$ & $\begin{array}{c}19.5647 \\
\pm \\
0.9893\end{array}$ \\
\hline $\begin{array}{l}\text { Total for } \\
\text { white } \\
\text { maize }\end{array}$ & $\begin{array}{c}363.5299 \\
\pm \\
18.4682\end{array}$ & $\begin{array}{c}124.1377 \\
\pm \\
19.1504\end{array}$ & $\begin{array}{c}1.9982 \\
\pm \\
0.0570\end{array}$ & $\begin{array}{c}74.6505 \\
\pm \\
5.3761\end{array}$ & $\begin{array}{c}55.2047 \\
\pm \\
1.8179\end{array}$ & $\begin{array}{c}80.7028 \\
\pm \\
0.5288\end{array}$ & $\begin{array}{c}1979.5072 \\
\pm \\
14.0324\end{array}$ & $\begin{array}{c}1.7080 \\
\pm \\
0.0340\end{array}$ & $\begin{array}{c}119.7166 \\
\pm \\
3.3413\end{array}$ & $\begin{array}{c}9.4731 \\
\pm \\
0.3689\end{array}$ & $\begin{array}{c}1.9411 \\
\pm \\
0.0712\end{array}$ & $\begin{array}{c}113.1811 \\
\pm \\
4.2564\end{array}$ \\
\hline
\end{tabular}

KEY: Values are mean $\pm S E, n=6$, key: $C / P=$ Crude Protein, $C / F a t=C r u d e$ fat, $C / F=$ crude fiber and c/glycosides: cyanogenic glycosides, $T / P=T o t a l$ phenol 
Table.2 Level of Mineral Elements ( $\mu \mathrm{g} / \mathrm{g})$ in the Recipes for TuwonMasara (white maize) with Kuka Soup) Consumed in Jigawa State Nigeria

\begin{tabular}{|c|c|c|c|c|c|c|c|c|c|c|c|c|c|c|}
\hline Sample & Se & Zn & Cd & Ni & $\mathbf{C u}$ & $\mathbf{P b}$ & Co & Mn & $\mathrm{Cr}$ & $\mathbf{C a}$ & Mg & $\mathbf{F e}$ & Ag & $\mathbf{K}$ \\
\hline $\begin{array}{l}\text { Maize } \\
\text { (white) }\end{array}$ & $\begin{array}{c}0.4789 \\
\pm \\
0.0131\end{array}$ & $\begin{array}{c}0.6520 \\
\pm \\
0.0325\end{array}$ & BDL & $\begin{array}{c}0.0784 \\
\pm \\
0.0064\end{array}$ & $\begin{array}{c}0.0842 \\
\pm \\
0.0205\end{array}$ & BDL & BDL & $\begin{array}{c}0.1791 \\
\pm \\
0.0314\end{array}$ & $\begin{array}{c}0.0178 \\
\pm \\
0.0003\end{array}$ & $\begin{array}{c}4.2105 \\
\pm \\
0.1996\end{array}$ & $\begin{array}{c}28.9455 \\
\pm \\
0.3434\end{array}$ & $\begin{array}{c}0.9123 \\
\pm \\
0.0555\end{array}$ & $\begin{array}{c}0.0155 \\
\pm \\
0.0004\end{array}$ & $\begin{array}{c}72.6225 \\
\pm \\
0.3642\end{array}$ \\
\hline Boabab & $\begin{array}{c}0.5063 \\
\pm \\
0.0456\end{array}$ & $\begin{array}{c}1.5439 \\
\pm \\
0.0921\end{array}$ & BDL & $\begin{array}{c}0.1493 \\
\pm \\
0.0070\end{array}$ & $\begin{array}{c}0.1434 \\
\pm \\
0.0151\end{array}$ & BDL & BDL & $\begin{array}{c}1.0082 \\
\pm \\
0.1392\end{array}$ & $\begin{array}{c}0.0704 \\
\pm \\
0.0035\end{array}$ & $\begin{array}{c}661.2982 \\
\pm \\
2.8376\end{array}$ & $\begin{array}{c}57.0838 \\
\pm \\
5.1297\end{array}$ & $\begin{array}{c}10.8880 \\
\pm \\
1.3322\end{array}$ & $\begin{array}{c}0.0133 \\
\pm \\
0.0013\end{array}$ & $\begin{array}{c}65.5710 \\
\pm \\
0.6966\end{array}$ \\
\hline Potash & $\begin{array}{c}0.0000 \\
\pm \\
0.0000\end{array}$ & $\begin{array}{c}4.0928 \\
\pm \\
0.0921\end{array}$ & BDL & $\begin{array}{c}0.2138 \\
\pm \\
0.0335\end{array}$ & $\begin{array}{c}0.0761 \\
\pm \\
0.0057\end{array}$ & BDL & BDL & $\begin{array}{c}4.8105 \\
\pm \\
0.0440\end{array}$ & $\begin{array}{c}0.5120 \\
\pm \\
0.0495\end{array}$ & $\begin{array}{c}408.5240 \\
\pm \\
4.3509\end{array}$ & $\begin{array}{c}174.2628 \\
\pm \\
0.4290\end{array}$ & $\begin{array}{c}132.1742 \\
\pm \\
1.3203\end{array}$ & $\begin{array}{c}0.0018 \\
\pm \\
0.0006\end{array}$ & BDL \\
\hline Onion & $\begin{array}{c}1.5199 \\
\pm \\
0.3354\end{array}$ & $\begin{array}{c}0.6497 \\
\pm \\
0.1107\end{array}$ & BDL & $\begin{array}{c}0.2853 \\
\pm \\
0.0436\end{array}$ & $\begin{array}{c}0.1377 \\
\pm \\
0.0317\end{array}$ & BDL & BDL & $\begin{array}{c}0.2888 \\
\pm \\
0.0760\end{array}$ & $\begin{array}{c}0.0227 \\
\pm \\
0.0034\end{array}$ & $\begin{array}{c}181.8690 \\
\pm \\
6.3246\end{array}$ & $\begin{array}{c}24.9952 \\
\pm \\
1.3774\end{array}$ & $\begin{array}{c}2.1493 \\
\pm \\
0.5728\end{array}$ & BDL & $\begin{array}{c}61.3767 \\
\pm \\
0.4773\end{array}$ \\
\hline Daddawa & $\begin{array}{c}0.5012 \\
\pm \\
0.3016\end{array}$ & $\begin{array}{c}2.1836 \\
\pm \\
0.0486\end{array}$ & BDL & $\begin{array}{c}0.1657 \\
\pm \\
0.0087\end{array}$ & $\begin{array}{c}0.5613 \\
\pm \\
0.0373\end{array}$ & BDL & BDL & $\begin{array}{c}3.4098 \\
\pm \\
0.2351\end{array}$ & $\begin{array}{c}0.0698 \\
\pm \\
0.0042\end{array}$ & $\begin{array}{c}631.9311 \\
\pm \\
8.4463\end{array}$ & $\begin{array}{c}90.4148 \\
\pm \\
1.1103\end{array}$ & $\begin{array}{c}17.5518 \\
\pm \\
1.8713\end{array}$ & $\begin{array}{c}0.0085 \\
\pm \\
0.0032\end{array}$ & $\begin{array}{c}95.0195 \\
\pm \\
1.2124\end{array}$ \\
\hline Meat & $\begin{array}{c}0.1646 \\
\pm \\
0.0544\end{array}$ & $\begin{array}{c}2.9572 \\
\pm \\
0.2436\end{array}$ & BDL & $\begin{array}{c}0.0762 \\
\pm \\
0.0064\end{array}$ & $\begin{array}{c}0.1590 \\
\pm \\
0.0444\end{array}$ & BDL & BDL & $\begin{array}{c}0.0475 \\
\pm \\
0.0052\end{array}$ & $\begin{array}{c}0.0187 \\
\pm \\
0.0004\end{array}$ & $\begin{array}{c}301.0400 \\
\pm \\
5.8093\end{array}$ & $\begin{array}{c}28.3320 \\
\pm \\
0.3789\end{array}$ & $\begin{array}{c}3.5587 \\
\pm \\
0.3362\end{array}$ & $\begin{array}{c}0.0103 \\
\pm \\
0.0009\end{array}$ & $\begin{array}{c}125.2333 \\
\pm \\
0.4598\end{array}$ \\
\hline $\begin{array}{c}\text { Hot } \\
\text { pepper }\end{array}$ & $\begin{array}{c}0.3036 \\
\pm \\
0.0420\end{array}$ & $\begin{array}{c}0.5492 \\
\pm \\
0.0546\end{array}$ & BDL & $\begin{array}{c}0.4742 \\
\pm \\
0.0589\end{array}$ & $\begin{array}{c}0.2856 \\
\pm \\
0.0286\end{array}$ & BDL & BDL & $\begin{array}{c}0.5089 \\
\pm \\
0.0392\end{array}$ & $\begin{array}{c}0.0525 \\
\pm \\
0.0075\end{array}$ & $\begin{array}{c}146.3503 \\
\pm \\
6.3335\end{array}$ & $\begin{array}{c}51.8990 \\
\pm \\
3.5832\end{array}$ & $\begin{array}{c}5.7959 \\
\pm \\
0.7722\end{array}$ & $\begin{array}{c}0.0060 \\
\pm \\
0.0018\end{array}$ & BDL \\
\hline Water & $\begin{array}{c}0.6535 \\
\pm \\
0.0367\end{array}$ & $\begin{array}{c}0.0698 \\
\pm \\
0.0036\end{array}$ & $\begin{array}{c}0.0139 \\
\pm \\
0.0059\end{array}$ & $\begin{array}{c}0.1382 \\
\pm \\
0.0833\end{array}$ & $\begin{array}{c}0.0469 \\
\pm \\
0.0041\end{array}$ & BDL & BDL & $\begin{array}{c}0.0318 \\
\pm \\
0.0038\end{array}$ & $\begin{array}{c}0.0590 \\
\pm \\
0.0039\end{array}$ & $\begin{array}{c}30.6490 \\
\pm \\
0.8918\end{array}$ & $\begin{array}{c}6.7720 \\
\pm \\
0.9311\end{array}$ & $\begin{array}{c}1.3908 \\
\pm \\
0.1275\end{array}$ & $\begin{array}{c}0.0055 \\
\pm \\
0.0006\end{array}$ & $\begin{array}{c}41.0318 \\
\pm \\
4.6184\end{array}$ \\
\hline $\begin{array}{l}\text { Total for } \\
\text { white } \\
\text { maize }\end{array}$ & $\begin{array}{c}4.1280 \\
\pm \\
0.8288\end{array}$ & $\begin{array}{c}12.6982 \\
\pm \\
0.7961\end{array}$ & $\begin{array}{c}0.0139 \\
\pm \\
0.0059\end{array}$ & $\begin{array}{c}1.5811 \\
\pm \\
0.2578\end{array}$ & $\begin{array}{c}1.4942 \\
\pm \\
0.1874\end{array}$ & BDL & BDL & $\begin{array}{c}10.2846 \\
\pm \\
0.5739\end{array}$ & $\begin{array}{c}0.8229 \\
\pm \\
0.0727\end{array}$ & $\begin{array}{c}2365.8721 \\
\pm \\
35.1936\end{array}$ & $\begin{array}{c}462.7051 \\
\pm \\
13.2830\end{array}$ & $\begin{array}{c}174.4210 \\
\pm \\
6.2880\end{array}$ & $\begin{array}{c}0.0609 \\
\pm \\
0.0088\end{array}$ & $\begin{array}{c}460.8548 \\
\pm \\
7.8287\end{array}$ \\
\hline
\end{tabular}

Values are mean \pm SE, $n=6, B D L=$ Below Detection Limit 
Table.3 Percentage $(\mathrm{g} / 100 \mathrm{~g})$ proximate and anti-nutritional content of recipe for danwake served with groundnut oil and pepper consumed in Jigawa state

\begin{tabular}{|c|c|c|c|c|c|c|c|c|c|c|c|c|}
\hline $\begin{array}{c}\text { Parameters } \\
\text { Sample }\end{array}$ & Carbohydrate & $\mathbf{C} / \mathbf{P}$ & C/Fat & $\mathrm{C} / \mathrm{F}$ & Moisture & Ash & $\begin{array}{c}\text { Energy } \\
\text { (kcal) }\end{array}$ & $\begin{array}{c}\text { C. } \\
\text { Glycosides } \\
(\mathbf{m g} / \mathbf{1 0 0 g})\end{array}$ & $\begin{array}{c}\text { Oxalate } \\
(\mathrm{mg} / \mathbf{1 0 0 g})\end{array}$ & Phytate & Tannin & $\mathbf{T} / \mathbf{P}$ \\
\hline Guinea corn & $\begin{array}{c}84.7872 \\
\pm \\
0.9806\end{array}$ & $\begin{array}{c}7.3889 \\
\pm \\
0.9614\end{array}$ & $\begin{array}{c}0.2761 \\
\pm \\
0.0057\end{array}$ & $\begin{array}{c}0.5142 \\
\pm \\
0.0057\end{array}$ & $\begin{array}{c}6.8333 \\
\pm \\
0.1667\end{array}$ & $\begin{array}{c}0.3336 \\
\pm \\
0.1667\end{array}$ & $\begin{array}{c}371.3333 \\
\pm \\
0.3333\end{array}$ & $\begin{array}{c}0.122 \\
\pm \\
0.001\end{array}$ & $\begin{array}{c}4.0333 \\
\pm \\
0.1940\end{array}$ & $\begin{array}{c}1.4774 \\
\pm \\
0.0622\end{array}$ & $\begin{array}{c}0.1492 \\
\pm \\
0.0007\end{array}$ & $\begin{array}{c}5.5221 \\
\pm \\
0.2387\end{array}$ \\
\hline Cassava & $\begin{array}{c}90.1854 \\
\pm \\
0.1846\end{array}$ & $\begin{array}{c}1.1278 \\
\pm \\
0.1746\end{array}$ & $\begin{array}{c}0.2747 \\
\pm \\
0.0027\end{array}$ & $\begin{array}{c}0.4975 \\
\pm \\
0.0312\end{array}$ & $\begin{array}{c}7.3333 \\
\pm \\
0.2108\end{array}$ & $\begin{array}{c}0.5479 \\
\pm \\
0.0290\end{array}$ & $\begin{array}{c}367.6667 \\
\pm \\
0.5578\end{array}$ & $\begin{array}{c}0.133 \\
\pm \\
0.001\end{array}$ & $\begin{array}{c}2.9700 \\
\pm \\
0.2028\end{array}$ & $\begin{array}{c}0.2823 \\
\pm \\
0.0202\end{array}$ & $\begin{array}{c}0.1535 \\
\pm \\
0.0011\end{array}$ & $\begin{array}{c}4.9696 \\
\pm \\
0.9829\end{array}$ \\
\hline Beans & $\begin{array}{c}29.7399 \\
\pm \\
3.4482\end{array}$ & $\begin{array}{c}61.0944 \\
\pm \\
3.3616\end{array}$ & $\begin{array}{c}0.2774 \\
\pm \\
0.0020\end{array}$ & $\begin{array}{c}1.3517 \\
\pm \\
0.2952\end{array}$ & $\begin{array}{c}6.8333 \\
\pm \\
0.1667\end{array}$ & $\begin{array}{c}0.8366 \\
\pm \\
0.0664\end{array}$ & $\begin{array}{c}366.0000 \\
\pm \\
1.2383\end{array}$ & $\begin{array}{c}0.124 \\
\pm \\
0.000\end{array}$ & $\begin{array}{c}7.0400 \\
\pm \\
0.2664\end{array}$ & $\begin{array}{c}0.7812 \\
\pm \\
0.0279\end{array}$ & $\begin{array}{c}0.3348 \\
\pm \\
0.0112\end{array}$ & $\begin{array}{c}3.7275 \\
\pm \\
0.3659\end{array}$ \\
\hline Baobab & $\begin{array}{c}63.0745 \\
\pm \\
2.7174\end{array}$ & $\begin{array}{c}17.6889 \\
\pm \\
2.3411\end{array}$ & $\begin{array}{c}0.2762 \\
\pm \\
0.0054\end{array}$ & $\begin{array}{c}10.4975 \\
\pm \\
0.7862\end{array}$ & $\begin{array}{c}6.3333 \\
\pm \\
0.4216\end{array}$ & $\begin{array}{c}2.2296 \\
\pm \\
0.0187\end{array}$ & $\begin{array}{c}325.6667 \\
\pm \\
3.1269\end{array}$ & $\begin{array}{c}0.422 \\
\pm \\
0.013\end{array}$ & $\begin{array}{c}7.8833 \\
\pm \\
0.0883\end{array}$ & $\begin{array}{c}1.6089 \\
\pm \\
0.0496\end{array}$ & $\begin{array}{c}0.5059 \\
\pm \\
0.0066\end{array}$ & $\begin{array}{c}36.8128 \\
\pm \\
1.0375\end{array}$ \\
\hline Pepper & $\begin{array}{c}42.6425 \\
\pm \\
1.8415\end{array}$ & $\begin{array}{c}16.7222 \\
\pm \\
1.5344\end{array}$ & $\begin{array}{c}0.3118 \\
\pm \\
0.0156\end{array}$ & $\begin{array}{c}33.0267 \\
\pm \\
1.0431\end{array}$ & $\begin{array}{c}4.5000 \\
\pm \\
0.2236\end{array}$ & $\begin{array}{c}2.8802 \\
\pm \\
0.5887\end{array}$ & $\begin{array}{c}240.3333 \\
\pm \\
3.252\end{array}$ & $\begin{array}{c}0.246 \\
\pm \\
0.006\end{array}$ & $\begin{array}{c}17.7100 \\
\pm \\
0.2588\end{array}$ & $\begin{array}{c}1.4194 \\
\pm \\
0.0430\end{array}$ & $\begin{array}{c}0.2909 \\
\pm \\
0.0046\end{array}$ & $\begin{array}{c}22.4932 \\
\pm \\
1.5294\end{array}$ \\
\hline Potash & $\begin{array}{c}17.4949 \\
\pm \\
0.1083\end{array}$ & $\begin{array}{c}1.6772 \\
\pm \\
0.0717\end{array}$ & $\begin{array}{c}0.2969 \\
\pm \\
0.0120\end{array}$ & $\begin{array}{c}0.6142 \\
\pm \\
0.0266\end{array}$ & $\begin{array}{c}6.3333 \\
\pm \\
0.3333\end{array}$ & $\begin{array}{c}73.7385 \\
\pm \\
0.2955\end{array}$ & $\begin{array}{c}79.3405 \\
\pm \\
0.8280\end{array}$ & $\begin{array}{c}0.113 \\
\pm \\
0.000\end{array}$ & $\begin{array}{c}0.9900 \\
\pm \\
0.0942\end{array}$ & $\begin{array}{c}0.4061 \\
\pm \\
0.0310\end{array}$ & $\begin{array}{c}0.0281 \\
\pm \\
0.0038\end{array}$ & $\begin{array}{c}0.3364 \\
\pm \\
0.0213\end{array}$ \\
\hline Total & $\begin{array}{c}327.9244 \\
\pm \\
9.2806\end{array}$ & $\begin{array}{c}105.6944 \\
\pm \\
8.4448\end{array}$ & $\begin{array}{c}1.7131 \\
\pm \\
0.1838\end{array}$ & $\begin{array}{c}46.5018 \\
\pm \\
2.2258\end{array}$ & $\begin{array}{c}38.1665 \\
\pm \\
1.5227\end{array}$ & $\begin{array}{c}80.5664 \\
\pm \\
1.0341\end{array}$ & $\begin{array}{c}1750.3405 \\
\pm \\
9.3366\end{array}$ & $\begin{array}{c}1.1610 \\
\pm \\
0.0210\end{array}$ & $\begin{array}{c}40.6266 \\
\pm \\
1.1045\end{array}$ & $\begin{array}{c}5.9753 \\
\pm \\
0.2339\end{array}$ & $\begin{array}{c}1.4624 \\
\pm \\
0.0280\end{array}$ & $\begin{array}{c}73.8616 \\
\pm \\
4.1757\end{array}$ \\
\hline
\end{tabular}

Values are mean $\pm \mathrm{SE}, \mathrm{n}=6$

Key: $\mathrm{C} / \mathrm{P}=$ crude protein, $\mathrm{c} / \mathrm{fat}=\mathrm{crude}$ fat, $\mathrm{c} / \mathrm{f}=$ =total fiber, $\mathrm{c} /$ glycosides $=$ Cyanogenic glycosides, $\mathrm{T} / \mathrm{P}=\mathrm{T}$ tal $\mathrm{phenol}$ 
Table.4 Level of mineral elements $(\mu \mathrm{g} / \mathrm{g})$ of recipe for Danwake with groundnut oil and pepper) consumed in Jigawa state, Nigeria

\begin{tabular}{|c|c|c|c|c|c|c|c|c|c|c|c|c|c|c|}
\hline Sample & Se & Zn & Cd & $\mathbf{N i}$ & $\mathrm{Cu}$ & $\mathbf{P b}$ & Co & Mn & $\mathrm{Cr}$ & $\mathrm{Ca}$ & Mg & $\mathbf{F e}$ & Ag & $\mathbf{K}$ \\
\hline $\begin{array}{l}\text { Guinea } \\
\text { corn }\end{array}$ & $\begin{array}{c}0.2841 \\
\pm \\
0.0134\end{array}$ & $\begin{array}{c}0.6090 \\
\pm \\
0.0201\end{array}$ & BDL & $\begin{array}{c}0.0600 \\
\pm \\
0.0068\end{array}$ & $\begin{array}{c}0.1122 \\
\pm \\
0.0038\end{array}$ & BDL & BDL & $\begin{array}{c}0.4881 \\
\pm \\
0.0065\end{array}$ & $\begin{array}{c}0.0341 \\
\pm \\
0.0014\end{array}$ & $\begin{array}{c}8.6530 \\
\pm \\
0.6564\end{array}$ & $\begin{array}{c}38.3393 \\
\pm \\
0.3157\end{array}$ & $\begin{array}{c}4.4325 \\
\pm \\
0.2537\end{array}$ & $\begin{array}{c}0.0132 \\
\pm \\
0.0002\end{array}$ & $\begin{array}{c}73.9953 \\
\pm \\
1.3073\end{array}$ \\
\hline Cassava & $\begin{array}{c}0.5762 \\
\pm \\
0.0578\end{array}$ & $\begin{array}{c}0.3137 \\
\pm \\
0.0285\end{array}$ & $\begin{array}{c}0.0019 \\
\pm \\
0.0009\end{array}$ & $\begin{array}{c}0.0702 \\
\pm \\
0.0046\end{array}$ & $\begin{array}{c}0.1190 \\
\pm \\
0.0048\end{array}$ & BDL & BDL & $\begin{array}{c}0.2497 \\
\pm \\
0.0169\end{array}$ & $\begin{array}{c}0.0343 \\
\pm \\
0.0076\end{array}$ & $\begin{array}{c}18.6892 \\
\pm \\
1.5371\end{array}$ & $\begin{array}{c}23.6612 \\
\pm \\
1.9141\end{array}$ & $\begin{array}{c}0.7903 \\
\pm \\
0.1381\end{array}$ & $\begin{array}{c}0.0150 \\
\pm \\
0.0006\end{array}$ & BDL \\
\hline Beans & $\begin{array}{c}0.5711 \\
\pm \\
0.0103\end{array}$ & $\begin{array}{c}0.9820 \\
\pm \\
0.0444\end{array}$ & BDL & $\begin{array}{c}0.2887 \\
\pm \\
0.0044\end{array}$ & $\begin{array}{c}0.3214 \\
\pm \\
0.0035\end{array}$ & BDL & BDL & $\begin{array}{c}0.7374 \\
\pm \\
0.0184\end{array}$ & $\begin{array}{c}0.0463 \\
\pm \\
0.0098\end{array}$ & $\begin{array}{c}23.9507 \\
\pm \\
0.9008\end{array}$ & $\begin{array}{c}55.8790 \\
\pm \\
2.3520\end{array}$ & $\begin{array}{c}3.3378 \\
\pm \\
0.0436\end{array}$ & $\begin{array}{c}0.0134 \\
\pm \\
0.0006\end{array}$ & BDL \\
\hline Boabab & $\begin{array}{c}0.5063 \\
\pm \\
0.0456\end{array}$ & $\begin{array}{c}1.5439 \\
\pm \\
0.2086\end{array}$ & BDL & $\begin{array}{c}0.1493 \\
\pm \\
0.0070\end{array}$ & $\begin{array}{c}0.1434 \\
\pm \\
0.0151\end{array}$ & BDL & BDL & $\begin{array}{c}1.0082 \\
\pm \\
0.1392\end{array}$ & $\begin{array}{c}0.0704 \\
\pm \\
0.0035\end{array}$ & $\begin{array}{c}661.2982 \\
\pm \\
2.8376\end{array}$ & $\begin{array}{c}57.0838 \\
\pm \\
5.1297\end{array}$ & $\begin{array}{c}10.8880 \\
\pm \\
1.3322\end{array}$ & $\begin{array}{c}0.0133 \\
\pm \\
0.0013\end{array}$ & $\begin{array}{c}65.5710 \\
\pm \\
0.6966\end{array}$ \\
\hline Pepper & $\begin{array}{c}0.5776 \\
\pm \\
0.0557\end{array}$ & $\begin{array}{c}0.4435 \\
\pm \\
0.0377\end{array}$ & BDL & $\begin{array}{c}0.1150 \\
\pm \\
0.0027\end{array}$ & $\begin{array}{c}0.3488 \\
\pm \\
0.0306\end{array}$ & BDL & BDL & $\begin{array}{c}0.4943 \\
\pm \\
0.0014\end{array}$ & $\begin{array}{c}0.0385 \\
\pm \\
0.0004\end{array}$ & $\begin{array}{c}29.3460 \\
\pm \\
2.6748\end{array}$ & $\begin{array}{c}42.8410 \\
\pm \\
1.7607\end{array}$ & $\begin{array}{c}6.3045 \\
\pm \\
0.1697\end{array}$ & $\begin{array}{c}0.0100 \\
\pm \\
0.0000\end{array}$ & BDL \\
\hline Potash & $\mathrm{BDL}$ & $\begin{array}{c}4.0928 \\
\pm \\
0.0921\end{array}$ & BDL & $\begin{array}{c}0.2138 \\
\pm \\
0.0335\end{array}$ & $\begin{array}{c}0.0761 \\
\pm \\
0.0057\end{array}$ & BDL & BDL & $\begin{array}{c}4.8105 \\
\pm \\
0.0440\end{array}$ & $\begin{array}{c}0.5120 \\
\pm \\
0.0495\end{array}$ & $\begin{array}{c}408.5240 \\
\pm \\
4.3509\end{array}$ & $\begin{array}{c}174.2628 \\
\pm \\
0.4290\end{array}$ & $\begin{array}{c}132.1742 \\
\pm \\
1.3203\end{array}$ & $\begin{array}{c}0.0018 \\
\pm \\
0.0006\end{array}$ & BDL \\
\hline Water & $\begin{array}{c}0.6535 \\
\pm \\
0.0367\end{array}$ & $\begin{array}{c}0.0698 \\
\pm \\
0.0036\end{array}$ & $\begin{array}{c}0.0139 \\
\pm \\
0.0059\end{array}$ & $\begin{array}{c}0.1382 \\
\pm \\
0.0833\end{array}$ & $\begin{array}{c}0.0469 \\
\pm \\
0.0041\end{array}$ & $\mathrm{BDL}$ & BDL & $\begin{array}{c}0.0318 \\
\pm \\
0.0038\end{array}$ & $\begin{array}{c}0.0590 \\
\pm \\
0.0039\end{array}$ & $\begin{array}{c}30.6490 \\
\pm \\
0.8918\end{array}$ & $\begin{array}{c}6.7720 \\
\pm \\
0.9311\end{array}$ & $\begin{array}{c}1.3908 \\
\pm \\
0.1275\end{array}$ & $\begin{array}{c}0.0055 \\
\pm \\
0.0006\end{array}$ & $\begin{array}{c}41.0318 \\
\pm \\
4.6184\end{array}$ \\
\hline Total & $\begin{array}{c}3.1688 \\
\pm \\
0.2195\end{array}$ & $\begin{array}{c}8.0547 \\
\pm \\
0.4350\end{array}$ & $\begin{array}{c}0.0158 \\
\pm \\
0.0068\end{array}$ & $\begin{array}{c}1.0352 \\
\pm \\
0.4230\end{array}$ & $\begin{array}{c}1.1678 \\
\pm \\
0.0676\end{array}$ & BDL & BDL & $\begin{array}{c}7.8200 \\
\pm \\
0.2302\end{array}$ & $\begin{array}{c}0.7946 \\
\pm \\
0.0761\end{array}$ & $\begin{array}{c}1181.1101 \\
\pm \\
13.8494\end{array}$ & $\begin{array}{c}398.8391 \\
\pm \\
12.8323\end{array}$ & $\begin{array}{c}159.3181 \\
\pm \\
3.3851\end{array}$ & $\begin{array}{c}0.0723 \\
\pm \\
0.0039\end{array}$ & $\begin{array}{c}180.5981 \\
\pm \\
6.6223\end{array}$ \\
\hline
\end{tabular}

Key: Values are mean $\pm \mathrm{SE}, \mathrm{n}=6, \mathrm{BDL}=$ BelowDetection Limit 
Table.5 Compares the percentage $(\mathrm{g} / 100 \mathrm{~g})$ total proximate and Anti Nutritional Content of Tuwon masara and Danwake Diets Consumed in Jigawa State, Nigeria

\begin{tabular}{|c|c|c|c|c|c|c|c|c|c|c|c|}
\hline $\begin{array}{l}\text { Parameters } \\
\text { Sample }\end{array}$ & $\begin{array}{c}\text { Carbo } \\
\text { hydrate }\end{array}$ & $\mathrm{C} / \mathrm{P}$ & C/Fat & $\mathrm{C} / \mathrm{F}$ & Moisture & Ash & $\begin{array}{c}\text { Energy } \\
\text { (kcal) }\end{array}$ & $\begin{array}{c}\text { C. } \\
\text { Glycosides } \\
(\mathbf{m g} / \mathbf{1 0 0 g})\end{array}$ & $\begin{array}{l}\text { Oxalate } \\
\text { (mg/100g) }\end{array}$ & Phytate & Tannin \\
\hline $\begin{array}{c}\text { Total for } \\
\text { white maize }\end{array}$ & $\begin{array}{c}363.5299 \\
\pm \\
18.4682\end{array}$ & $\begin{array}{c}124.1377 \\
\pm \\
19.1504\end{array}$ & $\begin{array}{c}1.9982 \\
\pm \\
0.0570\end{array}$ & $\begin{array}{c}74.6505 \\
\pm \\
5.3761\end{array}$ & $\begin{array}{c}55.2047 \\
\pm \\
1.8179\end{array}$ & $\begin{array}{c}80.7028 \\
\pm \\
0.5288\end{array}$ & $\begin{array}{c}1979.50 \\
\pm \\
14.032\end{array}$ & $\begin{array}{c}1.7080 \\
\pm \\
0.0340\end{array}$ & $\begin{array}{c}119.7166 \\
\pm \\
3.3413\end{array}$ & $\begin{array}{c}9.4731 \\
\pm \\
0.3689\end{array}$ & $\begin{array}{c}1.9411 \\
\pm \\
0.0712\end{array}$ \\
\hline $\begin{array}{c}\text { Total for } \\
\text { Danwake } \\
\text { with } \\
\text { groundnut } \\
\text { oil }\end{array}$ & $\begin{array}{c}327.9244 \\
\pm \\
9.2806\end{array}$ & $\begin{array}{c}105.6944 \\
\pm \\
8.4448\end{array}$ & $\begin{array}{c}1.7131 \\
\pm \\
0.1838\end{array}$ & $\begin{array}{c}46.5018 \\
\pm \\
2.2258\end{array}$ & $\begin{array}{c}38.1665 \\
\pm \\
1.5227\end{array}$ & $\begin{array}{c}80.5664 \\
\pm \\
1.0341\end{array}$ & $\begin{array}{c}1750.340 \\
\pm \\
9.3366\end{array}$ & $\begin{array}{c}1.1610 \\
\pm \\
0.0210\end{array}$ & $\begin{array}{c}40.6266 \\
\pm \\
1.1045\end{array}$ & $\begin{array}{c}5.9753 \\
\pm \\
0.2339\end{array}$ & $\begin{array}{c}1.4624 \\
\pm \\
0.0280\end{array}$ \\
\hline $\begin{array}{l}\text { RDA }(\mathrm{g} / \mathrm{d}) \\
\text { FAO } \\
\text { recommenda } \\
\text { tion }\end{array}$ & $60-120$ & 3.5 & $1.5-31.0$ & $19-38$ & $0.7-3.8$ & 0.8 & $\begin{array}{c}139 \\
-354\end{array}$ & NS & NS & NS & NS \\
\hline
\end{tabular}

Key: $\mathrm{C} / \mathrm{p}=$ crude protein, $\mathrm{C} / \mathrm{F}=$ crude fiber, $\mathrm{T} / \mathrm{P}=$ total phenol, NS=Not Stated

Table.6 Compares the level of mineral elements $(\mu \mathrm{g} / \mathrm{g})$ for the prepared selected diet (Tuwon masara and Danwake) consumed in Jigawa state, Nigeria

\begin{tabular}{|c|c|c|c|c|c|c|c|c|c|c|c|c|c|c|}
\hline Sample & Se & Zn & Cd & $\mathbf{N i}$ & $\mathrm{Cu}$ & $\mathbf{P b}$ & Co & Mn & $\mathrm{Cr}$ & $\mathbf{C a}$ & Mg & Fe & Ag & K \\
\hline $\begin{array}{l}\text { Total for } \\
\text { white } \\
\text { maize }\end{array}$ & $\begin{array}{c}4.1280 \\
\pm \\
0.8288\end{array}$ & $\begin{array}{c}12.6982 \\
\pm \\
0.7961\end{array}$ & $\begin{array}{c}0.0139 \\
\pm \\
0.0059\end{array}$ & $\begin{array}{c}1.5811 \\
\pm \\
0.2578\end{array}$ & $\begin{array}{c}1.4942 \\
\pm \\
0.1874\end{array}$ & BDL & BDL & $\begin{array}{c}10.2846 \\
\pm \\
0.5739\end{array}$ & $\begin{array}{c}0.8229 \\
\pm \\
0.0727\end{array}$ & $\begin{array}{c}2365.87 \\
\pm \\
35.19\end{array}$ & $\begin{array}{c}462.7051 \\
\pm \\
13.2830\end{array}$ & $\begin{array}{c}174.4210 \\
\pm \\
6.2880\end{array}$ & $\begin{array}{c}0.0609 \\
\pm \\
0.0088\end{array}$ & $\begin{array}{c}460.8548 \\
\pm \\
7.8287\end{array}$ \\
\hline $\begin{array}{l}\text { Total for } \\
\text { Danwake }\end{array}$ & $\begin{array}{c}3.1688 \\
\pm \\
0.2195\end{array}$ & $\begin{array}{c}8.0547 \\
\pm \\
0.4350\end{array}$ & $\begin{array}{c}0.0158 \\
\pm \\
0.0068\end{array}$ & $\begin{array}{c}1.0352 \\
\pm \\
0.4230\end{array}$ & $\begin{array}{c}1.1678 \\
\pm \\
0.0676\end{array}$ & BDL & BDL & $\begin{array}{c}7.8200 \\
\pm \\
0.2302\end{array}$ & $\begin{array}{c}0.7946 \\
\pm \\
0.0761\end{array}$ & $\begin{array}{c}1181.11 \\
\pm \\
13.84\end{array}$ & $\begin{array}{c}398.8391 \\
\pm \\
12.8323\end{array}$ & $\begin{array}{c}159.3181 \\
\pm \\
3.3851\end{array}$ & $\begin{array}{c}0.0723 \\
\pm \\
0.0039\end{array}$ & $\begin{array}{c}180.5981 \\
\pm \\
6.6223\end{array}$ \\
\hline $\begin{array}{l}\text { FAO } \\
\text { Recommen } \\
\text { dation } \\
(\mu \mathrm{g} / \mathrm{g})\end{array}$ & 6000 & 500 & NS & NS & 70 & NS & NS & $\begin{array}{l}1800- \\
2300\end{array}$ & $25-36$ & 12000 & 33000 & 1200 & NS & 117000 \\
\hline
\end{tabular}

Key: BDL=Below Detection Limit, NS=Not Stated 
Table 4 is the mineral element content $(\mu \mathrm{g} / \mathrm{g})$ of recipe for Danwake served with Groundnut Oil and pepper consumed in Jigawa State, Nigeria. Mineral elements analysed includes magnesium $(\mathrm{Mg})$, manganese $(\mathrm{Mn})$, iron $(\mathrm{Fe})$, lead $(\mathrm{Pb})$, Potassium (K) Calcium ( $\mathrm{Ca})$, Cupper (Cu), Cobalt (Co), Nickel (Ni), Cadmium (Cd), Zinc (Zn), selenium (Se), Chromium (Cr) and Argon (Ar).

Table 5 is the comparison of total percentage $(\mathrm{g} / 100 \mathrm{~g})$ proximate and Anti Nutritional Content of the two selected diets consumed in Jigawa State, Nigeria. The result indicates a high percentage of all the chemical compositions in Tuwon masara as compared to Danwake. The result also indicates that these two local foods have high chemical composition than the standard set by FAO.

Table 6 compared the level of Mineral Elements $(\mu \mathrm{g} / \mathrm{g})$ in the Recipes of the selected diet (Tuwon Masara with Kuka Soup and Danwake) Consumed in Jigawa State Nigeria. Content of some micro and macro elements such as magnesium $(\mathrm{Mg})$, manganese $(\mathrm{Mn})$, iron $(\mathrm{Fe})$, lead $(\mathrm{Pb})$, Potassium $(\mathrm{K})$ Calcium $(\mathrm{Ca})$, Cupper $(\mathrm{Cu})$, Cobalt $(\mathrm{Co})$, Nickel $(\mathrm{Ni})$, Cadmium (Cd), Zinc (Zn), selenium (Se), Chromium (Cr) and Argon (Ar) from these two local foods were compared with Dietary Recommended Allowance (DRA) as explain by Food and Agricultural Organization of the United Nation (FAO, 2010) standard.

\section{Discussion}

From this study, it was found that tuwon masara have more number of recipes which consist of white maize, baobab, potash, meat, onion, daddawa and hot pepper as compared to Danwake that consist of Guinea corn, cassava, potash, baobab and pepper as shown in table 1 and 2. Differences in recipes from these two traditional foods may proved a clear understanding that tuwon masara had high total proximate and anti-nutrient content of all parameters tested which includes carbohydrate, crude protein, crude fat, fiber, moisture, ash, energy, oxalates, cyanogenic glycosides, total phenol and tannin as compared to Danwake as indicated in table 5. These same of food prepared locally had high contents examined compared to Recommended Dietary Allowance (RDA) standard set aside by Food and Agricultural Organization (FAO) of the United Nation. These high contents in the diet can be attributed to procreate addition of recipes before, during and after processing which lead to micronutrient content of plant-based diets as reported by (Nnam, 2002; Mariam, 2005; Temple et al., 1996).

A diets consisting of cereals and legumes mixed with some animal protein source, have been reported to be sufficiently high in amino acids to meet RNI's (Recommended Nutrient Intakes) (FAO/WHO 1998). In table 2 for instance, tuwon masara contained 10 of the 13 mineral elements tested with only water having some amount of $\mathrm{Cd}$ while $\mathrm{Pb}$ and $\mathrm{Co}$ are below detection limit (BDL) in any of the recipes. For Danwake, similar situation occurred were $\mathrm{Pb}$ and $\mathrm{Co}$ are not detected from all the recipes tested but $\mathrm{Cd}$ and $\mathrm{K}$ are also only detected in some recipes which include water and cassava for $\mathrm{Cd}$ and Guinea corn, baobab and water for $\mathrm{K}$ respectively. Even though micronutrients are needed in very small quantities, they have tremendous impact on human health and wellbeing. Insufficient dietary intakes of these nutrients impair the functions of the brain, the immune and reproductive systems and energy metabolism. These deficiencies result in learning disabilities, reduced work capacity, serious illnesses, and death (Welch and Graham, 2002).

Differences in proximate and anti-nutritional contents of these two local foods can be seen 
in table 5 were tuwon masara had a high amount of all the nutrients in the recipes tested as compared to Danwake. Both local foods have high proximate contents as compared to standard in the Recommended Dietary Allowance set by FAO. The quality of these local foods may be affected by their high moisture contents.

Temple et al (1996) state that high moisture content in foods encourages microbial growth. This is necessary looking at the fact that local foods in Nigeria are prepared in high quantities and are normally kept for some time. It is in our view that local food be prepare in a small quantity and use at that particular time so as to avoid prolonged storage which in turn allow growth of pathogenic microbial flora (Mariam, 2005). The high fat contents of these local diets that are lower than RDA standard disagree with recommendations of $\mathrm{FAO} / \mathrm{WHO}$ (1998) that groundnut or vegetable oils are included in all foods, which will not only increase the energy density, but also be a transport vehicle for fat soluble vitamins.

With regards to mineral element contents, it was observed that Tuwon masara have high amount of all the micro and macro elements tested in this study with some mineral elements below detection limit. Compared to standard of Food and Agricultural Organization (FAO) of the United Nation, the mineral elements are found to be very small in quantity. Combs and Welch (1998) stated that, micronutrient deficiency may greatly increase mortality and morbidity rates, diminishes cognitive abilities of children and lowers their educational attainment. Therefore, an effort is needed to boast the quantity of mineral elements in local foods which is necessary as low mineral elements in foods may lower proper functioning and malnutrition as mentioned above.
In conclusion, this study revealed that these two local foods (Tuwon masara and Danwake) processed in Jigawa state Nigeria can meet some required nutrients for consumption since these nutritional contents are higher than the maximum requirements set aside by Food and Agricultural Organisation (FAO) and as such did not make a balanced diet. However, low micro and macro element contents in all the foods as compared to the RDA standard may lead to malnutrition. Therefore, fortification with appropriate micronutrients or micronutrientdense foodstuffs is necessary.

This research recommends that, proper methods on the amount of recipes that may contain high mineral elements will ultimately improve the quality of food there by preventing food related diseases like diabetes, obesity and malnutrition. This is believed to be a practical food-based approach aimed at combating the problem of malnutrition among Jigawa state populace in particular and Nigeria at large. Further study is in locally processed food is necessary so as to address the anti- nutritional factors, as well as the bioavailability of macro and micronutrients in an effort to provide food containing all the available nutrients in a balance proportion.

\section{Acknowledgement}

This project was fully funded by Tertiary Education Trust Fund (TETFUND), a parastatal of Federal Ministry of Education Abuja, Nigeria under Institutions Based Research (IBR) program. The authors also acknowledged the use of laboratory facilities from Jigawa state Polytechnic Dutse Nigeria.

\section{References} AOAC (1990): Official Methods of
$\begin{aligned} & \text { Analysis. } 15^{\text {th }} \\ & \text { efficial }\end{aligned}$ Association of
$\begin{aligned} & \text { Analytical } \\ & \text { Chemist., }\end{aligned}$ 
Washington D.C.

Combs G F, Jr and Welch R M (1998): Creating Healthful Food Systems: Linking Agriculture to Human Needs, Cornell International Institute for Food, Agriculture and Development, Ithaca, NY. 34

Gaffa, T., Jideani, I.A. and Nkama I. (2002): Traditional production, consumption and storage of Kunu - a non-alcoholic cereal beverage. Plant Foods for Human Nutrition, 57: 73-81.

Grusak, M.A. and Cakmak, I. (2005) Methods to improve the cropdelivery of minerals to humans and livestock. In Plant Nutritional Genomics (Broadley, M.R. and White, P. J., eds), 265-286, Blackwell

FAO/WHO (1998): Preparation and use of Food-Based Dietary Guidelines. Report of a Joint FAO/WHO Consultation. WHO Technical Report series 880 . Geneva.

Kikafunda, J.K., Abenakyo, L. and Lukwago, F.B. (2006): Nutritional and sensory properties of high energy/nutrient dense composite flour porridges from germinated maize and roasted beans for child-weaning in developing countries: a case for Uganda. Ecology Food Nutrition 45: 279-294.

Mariam S. (2005): Nutritive value of three potential complementary foods based on cereals and legumes; African Journal of Food and Nutritional Sciences; 5(2), 1-14.

National Population Commission (NPC, 2006): Population of Jigawa state Nigeria. www.jigawastate.gov.ng

Nnam NM (2002): Evaluation of
Complementary Foods based on Maize, Groundnut, Pawpaw and Mango Flour Blends. Nig. J. Nutr. Sci; 22(23): $8-18$.

Okafor, N. (1983): Processing of Nigerian indigenous fermented foods a chance for innovation. Nig Food J 1: 32-37

Temple, V. J., Badamosi E. J., Ladeji, O. and Solomon, M. (1996): Proximate Chemical Composition of three Locally Formulated Complementary Foods. West Afr. J. Biol. Sci. 5: 134 143.

Trichopoulou, A., Vasilopoulou, E., Georga. K., Soukara, S. and Dilis, V. (2006): Traditional foods: why and how to sustain them. Trends Food Sci Technol 17, 498-504.

Spackman, D. H. and Stein, E. H. (1958): Moore Automatic Recording Apparatus for use in the Chromatography of Amino acids. Analytical Chemistry; 30: 1190-1191.

UNICEF (2009): Tracking progress on childhood and maternal nutrition. UNICEF: New York

Welch, R. M. and Graham, R. D. (2002): Breeding crops for enhanced micronutrient content. Plant and Soil 245: 205-214

Welch, R.M. and Graham, R.D. (2002): Breeding for micronutrients in staple food crops from a human nutrition perspective. J. Exp. Bot. 55, 353-364

White, P. J. and Broadley, M. R. (2005): Biofortifying crops with essential mineral elements. TRENDS in Plant Science 10(12): 586-593. doi:10.1016/j.tplants.2005.10.001

\section{How to cite this article:}

Fatima Ibrahim Baiwa, Abdulhadi Yakubu, Garba Uba Kanya, Salisu Ahmed and Shuaib Nasir Halliru. 2019. Comparative Analysis of Food Composition and Mineral Element Levels of Two Locally Prepared Foods in Jigawa State Nigeria. Int.J.Curr.Microbiol.App.Sci. 8(01): 26462656. doi: https://doi.org/10.20546/ijcmas.2019.801.278 